\title{
Fast Spin-Orbit Qubit in an Indium Antimonide Nanowire
}

\author{
J. W. G. van den Berg, ${ }^{1}$ S. Nadj-Perge, ${ }^{1}$ V. S. Pribiag, ${ }^{1}$ S. R. Plissard ${ }^{2}$ \\ E. P. A. M. Bakkers, ${ }^{1,2}$ S. M. Frolov, ${ }^{1}$ and L. P. Kouwenhoven ${ }^{1}$ \\ ${ }^{1}$ Kavli Institute of Nanoscience, Delft University of Technology, 2600 GA Delft, The Netherlands \\ ${ }^{2}$ Department of Applied Physics, Eindhoven University of Technology, $5600 \mathrm{MB}$ Eindhoven, The Netherlands
} (Received 13 October 2012; published 8 February 2013)

\begin{abstract}
Because of the strong spin-orbit interaction in indium antimonide, orbital motion and spin are no longer separated. This enables fast manipulation of qubit states by means of microwave electric fields. We report Rabi oscillation frequencies exceeding $100 \mathrm{MHz}$ for spin-orbit qubits in InSb nanowires. Individual qubits can be selectively addressed due to intrinsic differences in their $g$ factors. Based on Ramsey fringe measurements, we extract a coherence time $T_{2}^{*}=8 \pm 1 \mathrm{~ns}$ at a driving frequency of $18.65 \mathrm{GHz}$. Applying a Hahn echo sequence extends this coherence time to $34 \mathrm{~ns}$.
\end{abstract}

The spin of a single electron forms a two-level system, which makes it a natural choice for creating a quantum bit (qubit) [1]. Quantum information processing based on such qubits has developed into a mature and diverse field [2]. Previous work has demonstrated important milestones, including single-shot detection of spin state, coherent control of a single spin, and coherent coupling between two spins [2-6]. Of great importance for future development of spin-based quantum computation is combining efficient single-qubit control and two-qubit operations in the same system [7] and developing ways to integrate spin qubits with other quantum computing architectures. To pursue these goals, several promising material platforms are being explored. Among these are narrow band-gap semiconductor nanowires, such as indium arsenide and indium antimonide. This class of materials has recently gained considerable attention, due to their strong spin-orbit coupling, which enables efficient all-electrical spin control [5,8-11] and could provide a means of coupling qubits to quantum systems based on superconducting cavities [12].

In this Letter, we demonstrate an electrically controlled spin-orbit qubit in an indium antimonide nanowire. We observe Rabi oscillations with frequencies up to $104 \mathrm{MHz}$, the fastest reported to date for an electrically controlled single-spin qubit in a quantum dot. Furthermore, we show that the individual qubits in the two dots can be addressed with high selectivity, owing to a large $g$-factor difference between two dots. We achieve universal qubit control and study qubit coherence by means of Ramsey-type measurements. We find that the inhomogeneous dephasing time $T_{2}^{*}$ can be extended to $\sim 35$ ns by using a Hahn echo.

To realize our spin-orbit qubit, a double quantum dot is defined inside the nanowire by means of local electrostatic gating. The qubits' basis states are spin-orbit doublets (denoted by $\Uparrow$ and $\Downarrow$ ), which-analogous to conventional spin qubits-are split by the Zeeman energy in a magnetic field. Transitions between these states can be induced by applying microwave frequency electric fields.
An image of our device obtained by scanning electron microscopy is presented in Fig. 1(a). It consists of an indium antimonide nanowire $(\sim 1.5 \mu \mathrm{m}$ long, $100 \mathrm{~nm}$ thick) contacted by $\mathrm{Ti} / \mathrm{Al}$ source and drain electrodes. Below the nanowire, separated by a layer of $\mathrm{Si}_{3} \mathrm{~N}_{4}$ dielectric, is a set of five narrow gates $(60 \mathrm{~nm}$ pitch) used to induce a double quantum dot potential in the nanowire and control the number of electrons in these dots. Underneath another layer of $\mathrm{Si}_{3} \mathrm{~N}_{4}$ is a large metallic gate (BG) by which the conductance of the entire wire can be tuned. Measurements are performed in a ${ }^{3} \mathrm{He}$ system with a base temperature of $260 \mathrm{mK}$.

In order to read out and initialize our qubits, we take advantage of Pauli spin blockade [2,13]. When a bias voltage is applied between the source and drain of the double dot, sequential transport through the dots is possible in a triangular region in gate space. However, spin conservation introduces additional constraints on interdot tunneling. Although a transition may be energetically allowed, it may be prohibited by spin selection rules. For example, a triplet state with one electron in each dot such as $(\Uparrow, \Uparrow)$ cannot transition to a $(0,2)$ singlet state, unless one of the spin-orbit states is rotated. The current through the dots thus becomes suppressed, as eventually such a parallel triplet state is loaded. This principle is illustrated in Fig. 1(c). Note that the antiparallel $T_{0}$ state is in practice not blocked, as hyperfine interaction makes it quickly decay into a singlet state [2]. Rotation of a spin-orbit state from $\Uparrow$ to $\Downarrow$, or vice versa, can be accomplished by electricdipole spin resonance (EDSR). Application of an alternating electric field, resonant with the Larmor precession frequency, drives transitions between the spin-orbit states [14-17], lifting the spin blockade. To detect the EDSR, we measure the spin blockade leakage current as a function of applied magnetic field $B$ and microwave frequency $f$ [Fig. 1(b)]. From the slope of the resonance we extract a Landé $g$ factor of 41 for our quantum dot. In addition to the resonance, we observe a finite current around $B=0$ [18], 

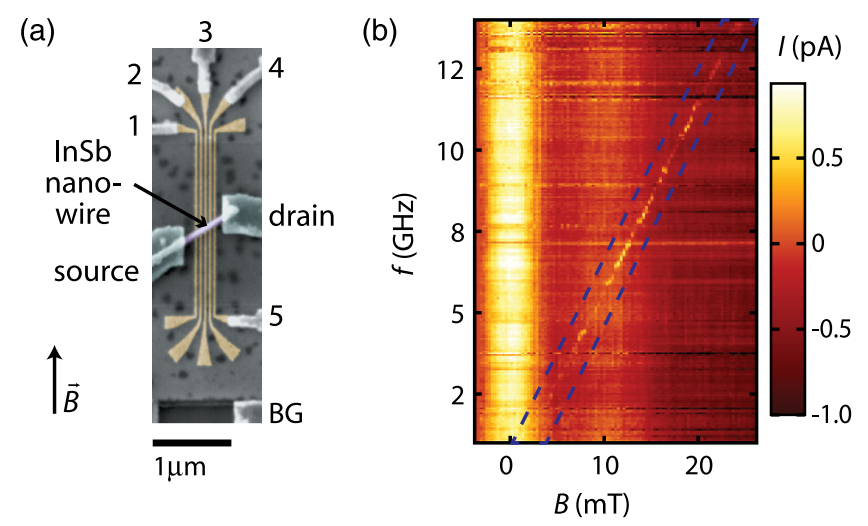

(c)

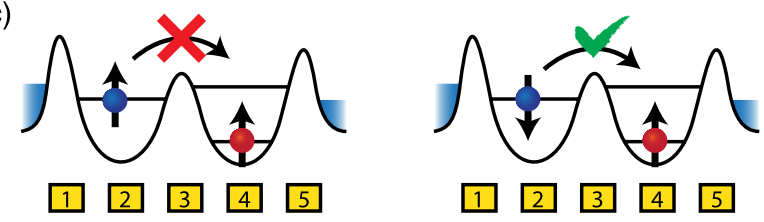

FIG. 1 (color online). (a) Electron microscope image of the device consisting of an InSb nanowire contacted by source and drain electrodes, lying across a set of fine gates (numbered, $60 \mathrm{~nm}$ pitch) as well as a larger bottom gate (labeled BG). (b) Current through the device when applying microwaves with the double dot in spin blockade configuration (a vertical linecut near $0 \mathrm{mT}$ has been subtracted to suppress resonances at constant frequency). When the microwave frequency matches the Larmor frequency (resonance highlighted by the dashed blue box), blockade is lifted and current increases. (c) Schematic illustration of Pauli spin blockade on which readout depends. Only antiparallel states (right) can occupy the same dot, allowing current through the device. A parallel configuration (left) leads to a suppression of the current.

resulting from mixing of the $(1,1)$ triplet states to the singlet by the nuclear field $[19,20]$.

To demonstrate coherent control over the qubit, we apply microwave bursts of variable length. First, the qubit is initialized into a spin-blocked charge configuration. This is accomplished by idling inside the bias triangle [Fig. 2(a)]. In order to prevent the electron from tunneling out of the dot during its subsequent manipulation, the double dot is maintained in Coulomb blockade in the same charge configuration. While in the Coulomb blockade regime, a microwave burst is applied. The double dot is then again quickly brought back to the spin blockade configuration by pulsing the plunger gates. By applying such microwave bursts [schematically depicted in Fig. 2(b)], we perform a Rabi measurement. If the manipulation has flipped the electron spin-orbit state, the blockade is lifted and an electron can move from the first to the second dot and exit again through the outgoing lead. By continuously repeating the pulse sequence and measuring the (dc) current through the double dot, we measure the Rabi oscillations associated with the rotation of the spin-orbit state [Fig. 2(c)].

We have achieved Rabi oscillations with more than five oscillation periods at a maximum frequency of $104 \mathrm{MHz}$.

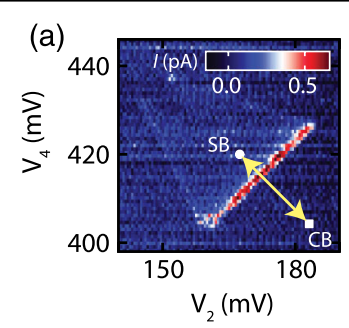

(b) initialize manipulate read-out

(c)

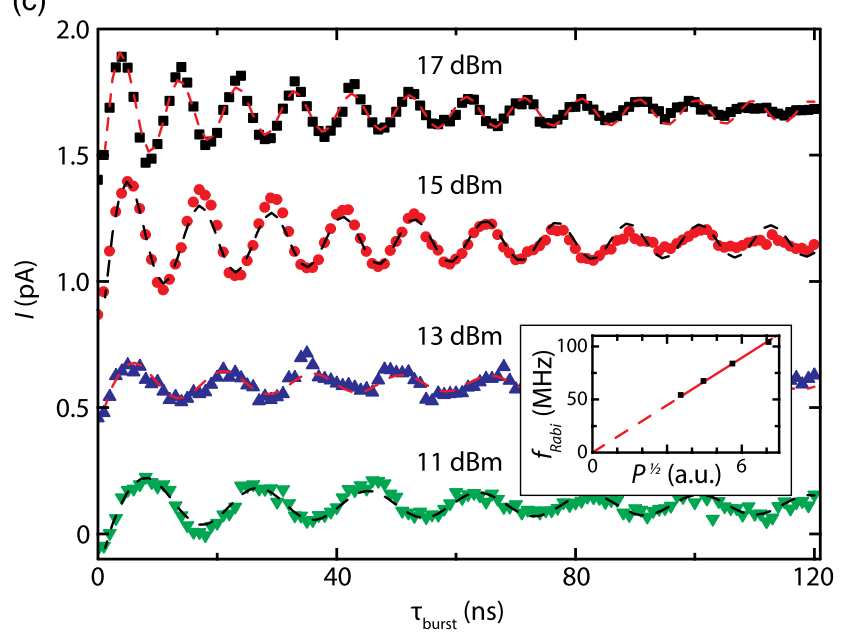

FIG. 2 (color online). (a) Bias triangle in which spin blockade was observed for a negative bias of $-5 \mathrm{mV}$. This is the $(2 m+1,2 n+1) \rightarrow(2 m, 2 n+2)$ transition (transition $A$; see Ref. [21]). (b) Sequence used for measuring Rabi oscillations. Pulses are applied to gates 2 and 4 to move the double dot along the detuning axis between Coulomb blockade (CB) and spin blockade (SB) configurations. In $\mathrm{CB}$ a microwave burst is applied via gate $B G$ to rotate the spin. (c) Rabi oscillation obtained at a magnetic field of $31.4 \mathrm{mT}$, driving frequency of $18.65 \mathrm{GHz}$, and source power of 11 (bottom) to $17 \mathrm{dBm}$ (top). Dashed lines are fits to $a \cos \left(f_{R} \tau_{\text {burst }}+\phi\right) \tau_{\text {burst }}^{-d}+b$, giving Rabi frequencies $f_{R}$ of $54 \pm 1,67 \pm 1,84 \pm 1$, and $104 \pm$ $1 \mathrm{MHz}$. Linear slopes, attributed to photon-assisted tunneling, of $0.5,0.6,0.4$, and $0.6 \mathrm{fA} / \mathrm{ns}$ (top to bottom) were subtracted. $d=0.5$ for the bottom trace and 0.4 for the others. Curves are offset by $0.5 \mathrm{pA}$ for clarity. Inset: Rabi frequencies set out against driving amplitudes, including a linear fit through 0 .

Even higher Rabi frequencies could be observed for larger microwave driving power [21], but this resulted in a quick damping of the oscillations, likely due to photon-assisted tunneling [22,23]. The high frequency allows for fast operation of the qubit, which is important for quantum information processing. Also important in this respect is the qubit manipulation fidelity. We can estimate this fidelity from the strength of the spin-orbit field relative to the fluctuations of the nuclear spin bath (believed to be the main source of dephasing) [22]. From the Rabi frequency of $104 \mathrm{MHz}$ we obtain a spin-orbit field $B_{\mathrm{SO}}=0.36 \mathrm{mT}$ driving the rotations. By taking the width of the EDSR peak as a measure for the nuclear magnetic field, we can estimate a value of $B_{N}=0.16 \pm 0.02 \mathrm{mT}$ for this field. 
With these values of $B_{N}$ and $B_{\mathrm{SO}}$ we estimate our qubit manipulation fidelity to be $81 \pm 6 \%$ [21]. We also note that the Rabi frequency is expected to be largest when $B_{\text {SO }}$ and $B$ are perpendicular [15]. Even faster Rabi frequencies are thus expected upon optimization of the magnetic field angle (currently the magnetic field is applied at an angle of $\sim 55^{\circ}$ to the nanowire), which would be an interesting topic for further study.

The Rabi experiment demonstrates rotation of the qubit around a single axis. However, in order to be able to prepare the qubit in any arbitrary superposition, it is necessary to achieve rotations around two independent axes. We demonstrate such universal control by means of a Ramsey experiment, where the axis of qubit rotation is determined by varying the phase of the applied microwave bursts, as illustrated at the top of Fig. 3(a). As in the Rabi experiment, the microwave bursts are applied while the dots are kept in Coulomb blockade, to maintain a well defined charge state and prevent the electrons from tunneling out during manipulation. In the Ramsey sequence an initial microwave burst rotates the state by $\pi / 2$ to the $x y$ plane of the Bloch sphere. We take this rotation axis to be the $x$ axis. A second burst is then applied after some delay $\tau$, making a $3 \pi / 2$ rotation. By varying the phase of this
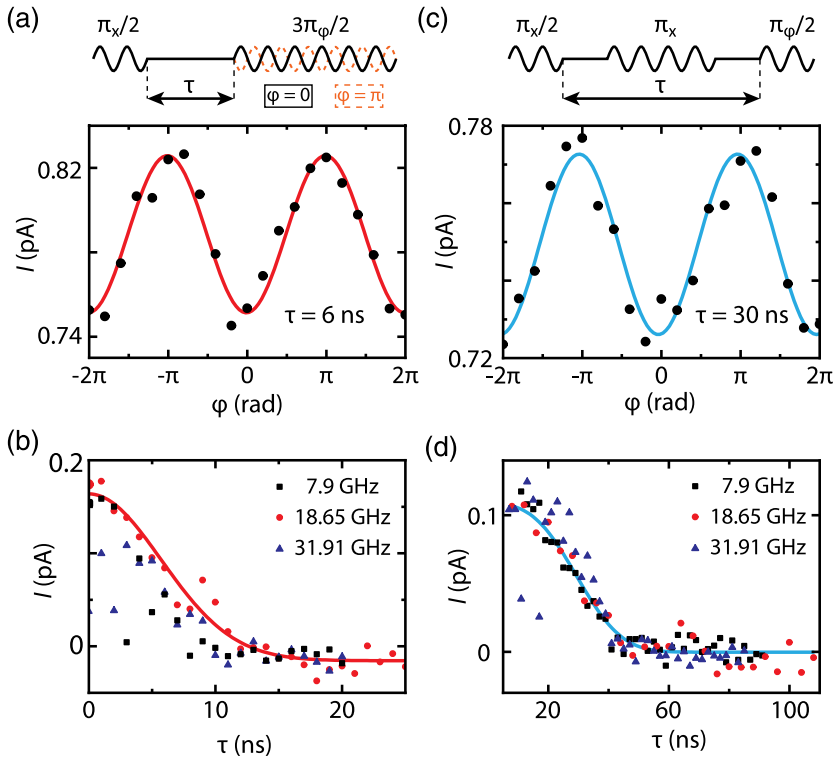

FIG. 3 (color online). (a) Ramsey experiment; an initial $\pi / 2$ pulse rotates the spin to the $x y$ plane. After some delay a $3 \pi / 2$ pulse is applied, restoring spin blockade or (partially) lifting it, depending on the phase of the pulse. (b) Decay of the Ramsey fringe contrast with increasing delay time $\tau$ for different driving frequencies. The solid line is a fit to $\exp \left[-\left(\tau / T_{2}^{*}\right)^{2}\right]$ at a driving frequency of $18.65 \mathrm{GHz}$, giving $T_{2}^{*}=8 \pm 1 \mathrm{~ns}$. (c) A Hahn echo sequence (top) extends the decay of the fringe contrast, to $30 \mathrm{~ns}$ in this case. (d) Decay of the fringe contrast in the Hahn echo sequence for different microwave frequencies. The solid line is a fit to $\exp \left[-\left(\tau / T_{\text {echo }}\right)^{3}\right]$ for driving frequency $18.65 \mathrm{GHz}$, yielding $T_{\text {echo }}=34 \pm 2 \mathrm{~ns}$. pulse with respect to the initial $\pi / 2$ pulse, we can control the axis of the second rotation [see Fig. 3(a)]. For example, if the two bursts are applied with the same phase, in total a $2 \pi$ rotation will have been made. This restores a spin blockade configuration, thus leading to a suppression of the current. A second burst with a phase $\pi$, however, would rotate the qubit in the opposite direction, ending up along the $|-z\rangle$ direction on the Bloch sphere. For this case spin blockade is thus fully lifted, and current increases to a maximum.

When the delay time between the first and final pulses in the Ramsey sequence is increased, the qubit starts to dephase. The loss of phase coherence leads to decay of the Ramsey fringe contrast, as shown in Fig. 3(b). By fitting the experimental data to $\exp \left[-\left(\tau / T_{2}^{*}\right)^{2}\right]$, we extract a dephasing time of $T_{2}^{*}=8 \pm 1 \mathrm{~ns}$, obtained at a driving frequency of $18.65 \mathrm{GHz}$. Other driving frequencies of 7.9 and $31.91 \mathrm{GHz}$ resulted in similar $T_{2}^{*}$ values of $6 \pm 1$ and $9 \pm 1 \mathrm{~ns}$, respectively. To extend the coherence of the qubit, we employ a Hahn echo technique [24,25]: Halfway between two $\pi / 2$ pulses, an extra pulse is applied to flip the state over an angle $\pi$. Doing so partially refocuses the dephasing caused by the nuclear magnetic field, which varies slowly compared to the electron spin dynamics [26]. From Fig. 3(c), where the total delay has been extended to $\tau=30 \mathrm{~ns}$, it is clear that this technique can maintain contrast of the Ramsey fringes for considerably longer times. An increase in the coherence time to $T_{\text {echo }}=34 \pm 2 \mathrm{~ns}$ is obtained from the decay of the contrast [Fig. 3(d)] for a driving frequency of $18.65 \mathrm{GHz}$. Similar values of $32 \pm 1$ and $35 \pm 1 \mathrm{~ns}$ were obtained at driving frequencies 7.9 and $31.91 \mathrm{GHz}$, respectively.

The relatively low coherence times obtained here are in line with previous results obtained for InAs nanowires [11]. The time scales show no significant dependence on driving frequency within the accessible range of $8-32 \mathrm{GHz}$. These results suggest the existence of a fast spin bath interacting with the electron, likely originating from the large nuclear spins of indium antimonide $\left(5 / 2\right.$ and $7 / 2$ for ${ }^{121} \mathrm{Sb}$ and ${ }^{123} \mathrm{Sb}$, respectively, and $9 / 2$ for In). It must be noted, though, that other sources of dephasing, such as nearby paramagnetic impurities or charge noise, cannot be completely ruled out.

Thus far we have presented only coherent rotations of one of the qubits. It is possible to individually address each qubit in the two dots if the EDSR peak splits into two separate resonances. This splitting can arise due to a $g$-factor difference between the two dots, as in our present device, but can also be engineered, e.g., through incorporation of a micromagnet into a device [17,27]. The main panel of Fig. 4 displays the two EDSR peaks corresponding to the two dots. From the magnetic field dependence of the resonances, we determine $g$ factors of 48 and 36 for the two dots, respectively [21]. This difference in $g$ factors for the two dots can be explained by a difference in confinement 


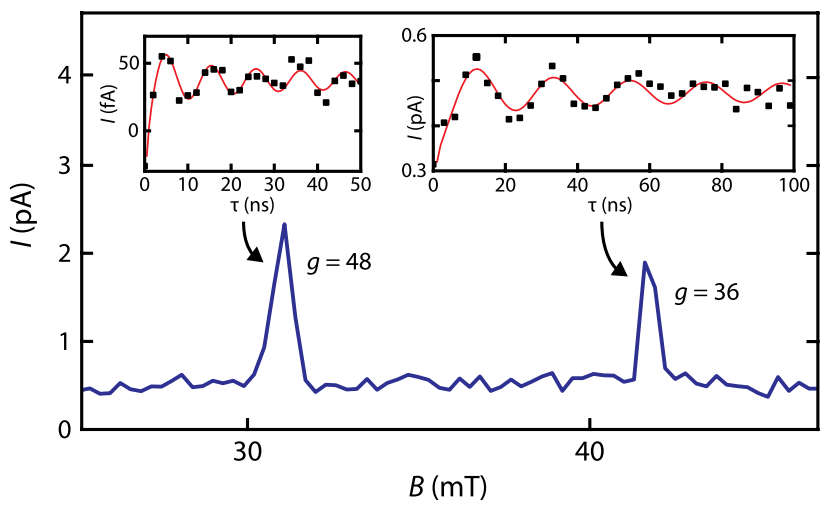

FIG. 4 (color online). Main panel: Two well separated EDSR peaks for the spin-orbit qubit in each of the two dots. The microwave driving frequency is $20.9 \mathrm{GHz}$. Insets: Rabi oscillations for the corresponding EDSR peaks. Linear slopes (attributed to photon-assisted tunneling) of 0.6 and $0.9 \mathrm{fA} / \mathrm{ns}$, respectively, are subtracted to flatten the average. The Rabi data on the left were obtained at $31.2 \mathrm{mT} B$ field and $20.9 \mathrm{GHz}$ driving frequency. From the fit (as in Fig. 2) a Rabi frequency of $96 \pm 2 \mathrm{MHz}$ is obtained. On the right, the field was $41.2 \mathrm{mT}$ and driving frequency $21 \mathrm{GHz}$. The Rabi frequency obtained from the fit is $47 \pm 3 \mathrm{MHz}$.

$[11,20,28,29]$. Note that these measurements were obtained at the charge transition $(2 m+1,2 n+3) \rightarrow$ $(2 m+2,2 n+2)$ (transition $B$ [21]) in opposite bias than the previously presented data. No second resonance was resolved for charge transition $A$, perhaps due to strongly similar $g$ factors or decreased coupling of the microwaves to the second dot.

Utilizing the large difference in $g$ factors between the two dots, we have achieved coherent control of both qubits. Here, we probe the qubits by using similar microwave frequencies but different magnetic fields. The insets in Fig. 4 show the Rabi oscillations obtained for each of the qubits. The frequency of the Rabi oscillations (see the insets in Fig. 4) for the qubit corresponding to $g$ factor 48, was $96 \mathrm{MHz}$. For the other dot, with a corresponding $g$ factor of 36, a lower Rabi frequency of $47 \mathrm{MHz}$ was achieved. This slower Rabi oscillation is consistent with a weaker coupling of the microwave electric field to this dot [30].

In summary, we have demonstrated the realization of a spin-orbit qubit in an indium antimonide nanowire. Fast manipulation and universal control of the qubit were demonstrated by Rabi and Ramsey measurements. A significant improvement in terms of speed and fidelity was achieved compared to previously realized indium arsenide spin-orbit qubits. Dephasing times, however, remained similar to InAs, suggesting that the nuclear spin bath is the main source of dephasing. The large interdot $g$-factor differences make possible the selective addressing of different qubits. Importantly, we estimate that the large interdot Zeeman energy difference would be sufficient to implement a fast $(\sim 1 \mathrm{~ns})$ two-qubit CPHASE gate [31,32], operating well within the $T_{\text {echo }}$ coherence time.
We thank V.E. Calado and M. Shafiei for discussions. Financial support for this work was given by ERC and The Netherlands Organisation for Scientific Research NWO/FOM.

[1] D. Loss and D. P. DiVincenzo, Phys. Rev. A 57, 120 (1998).

[2] R. Hanson, J.R. Petta, S. Tarucha, and L.M. K. Vandersypen, Rev. Mod. Phys. 79, 1217 (2007).

[3] J. R. Petta, A.C. Johnson, J. M. Taylor, E. A. Laird, A. Yacoby, M. D. Lukin, C. M. Marcus, M. P. Hanson, and A. C. Gossard, Science 309, 2180 (2005).

[4] C. B. Simmons, J. R. Prance, B. J. Van Bael, T. S. Koh, Z. Shi, D. E. Savage, M. G. Lagally, R. Joynt, M. Friesen, S. N. Coppersmith, and M. A. Eriksson, Phys. Rev. Lett. 106, 156804 (2011).

[5] S. Nadj-Perge, S. M. Frolov, J.W.W. van Tilburg, J. Danon, Y. V. Nazarov, R.E. Algra, E.P. A. M. Bakkers, and L. P. Kouwenhoven, Phys. Rev. B 81, 201305 (2010).

[6] B. M. Maune, M. G. Borselli, B. Huang, T. D. Ladd, P. W. Deelman, K. S. Holabird, A. A. Kiselev, I. AlvaradoRodriguez, R. S. Ross, A. E. Schmitz, M. Sokolich, C. A. Watson, M. F. Gyure, and A. T. Hunter, Nature (London) 481, 344 (2012).

[7] M. D. Shulman, O.E. Dial, S. P. Harvey, H. Bluhm, V. Umansky, and A. Yacoby, Science 336, 202 (2012).

[8] M. T. Björk, A. Fuhrer, A. E. Hansen, M. W. Larsson, L. E. Fröberg, and L. Samuelson, Phys. Rev. B 72, 201307 (2005).

[9] A. Pfund, I. Shorubalko, K. Ensslin, and R. Leturcq, Phys. Rev. B 76, 161308 (2007).

[10] H. A. Nilsson, P. Caroff, C. Thelander, M. Larsson, J. B. Wagner, L.-E. Wernersson, L. Samuelson, and H. Q. Xu, Nano Lett. 9, 3151 (2009).

[11] S. Nadj-Perge, S. M. Frolov, E. P. A. M. Bakkers, and L.P. Kouwenhoven, Nature (London) 468, 1084 (2010).

[12] K. D. Petersson, L. W. McFaul, M. D. Schroer, M. Jung, J. M. Taylor, A. A. Houck, and J.R. Petta, Nature (London) 490, 380 (2012).

[13] A. Pfund, I. Shorubalko, K. Ensslin, and R. Leturcq, Phys. Rev. Lett. 99, 036801 (2007).

[14] E. I. Rashba and A. L. Efros, Phys. Rev. Lett. 91, 126405 (2003).

[15] V. N. Golovach, M. Borhani, and D. Loss, Phys. Rev. B 74, 165319 (2006).

[16] E. A. Laird, C. Barthel, E. I. Rashba, C. M. Marcus, M. P. Hanson, and A.C. Gossard, Phys. Rev. Lett. 99, 246601 (2007).

[17] M. Pioro-Ladrière, T. Obata, Y. Tokura, Y.-S. Shin, T. Kubo, K. Yoshida, T. Taniyama, and S. Tarucha, Nat. Phys. 4, 776 (2008).

[18] Because of a small offset in the magnet power supply output, the magnetic field scale has been offset to have 0 coincide with the hyperfine peak.

[19] F. H. L. Koppens, J. A. Folk, J. M. Elzerman, R. Hanson, L. H. W. van Beveren, I. T. Vink, H.P. Tranitz, W. Wegscheider, L.P. Kouwenhoven, and L. M. K. Vandersypen, Science 309, 1346 (2005). 
[20] S. Nadj-Perge, V. S. Pribiag, J. W. G. van den Berg, K. Zuo, S. R. Plissard, E. P. A. M. Bakkers, S. M. Frolov, and L. P. Kouwenhoven, Phys. Rev. Lett. 108, 166801 (2012).

[21] See Supplemental Material at http://link.aps.org/ supplemental/10.1103/PhysRevLett.110.066806 for details on double dot occupation, faster Rabi oscillations, nuclear field and fidelity estimates, and individual addressing of the qubits.

[22] F. H.L. Koppens, C. Buizert, K. J. Tielrooij, I. T. Vink, K. C. Nowack, T. Meunier, L.P. Kouwenhoven, and L. M. K. Vandersypen, Nature (London) 442, 766 (2006).

[23] K. C. Nowack, F. H.L. Koppens, Y. V. Nazarov, and L. M. K. Vandersypen, Science 318, 1430 (2007).

[24] B. Herzog and E. Hahn, Phys. Rev. 103, 148 (1956).

[25] F. H. L. Koppens, K. C. Nowack, and L. M. K. Vandersypen, Phys. Rev. Lett. 100, 236802 (2008).
[26] D. J. Reilly, J. M. Taylor, E. A. Laird, J. R. Petta, C. M. Marcus, M. P. Hanson, and A. C. Gossard, Phys. Rev. Lett. 101, 236803 (2008).

[27] Y. Tokura, W. G. van der Wiel, T. Obata, and S. Tarucha, Phys. Rev. Lett. 96, 047202 (2006).

[28] C. E. Pryor and M.E. Flatté, Phys. Rev. Lett. 96, 026804 (2006).

[29] M. D. Schroer, K. D. Petersson, M. Jung, and J. R. Petta, Phys. Rev. Lett. 107, 176811 (2011).

[30] The Rabi for the $g=36$ dot was achieved at different gate voltages than for the other dot, to improve its visibility, though care was taken to measure the intended resonance.

[31] G. Burkard, D. Loss, D. P. DiVincenzo, and J. A. Smolin, Phys. Rev. B 60, 11404 (1999).

[32] T. Meunier, V. E. Calado, and L. M. K. Vandersypen, Phys. Rev. B 83, 121403 (2011). 